Long- term pot ent $i$ at $i$ on of neur onal exci tat $i$ on by neur on- $g$ l i a i nt eracti ons i $n$ the $r$ at spi nal dor sal hor $n$

\begin{tabular}{|l|l|}
\hline 著者 & $\begin{array}{l}\text { I KEDA H r oshi, TSUDA Nakot o, I NOUE Kazuhi de, } \\
\text { MRASE Kazuyuki }\end{array}$ \\
\hline $\begin{array}{l}\text { j our nal or } \\
\text { publ i cat i on titl e }\end{array}$ & Eur opean Journal of Neur osci ence \\
\hline vol une & 25 \\
\hline number & 5 \\
\hline page r ange & $1297-1306$ \\
\hline year & $2007-03$ \\
\hline URL & ht t p: //hdl . handl e. net /10098/1861 \\
\hline
\end{tabular}




\section{Long-term potentiation of neuronal excitation by neuron-glia interactions in the rat spinal dorsal horn}

Hiroshi Ikeda ${ }^{1}$, Makoto Tsuda ${ }^{2}$, Kazuhide Inoue ${ }^{2}$, Kazuyuki Murase ${ }^{1}$

${ }^{1}$ Department of Human and Artificial Intelligence Systems, Graduate School of

Engineering, and Research and Education Program for Life Science, University of Fukui,

3-9-1 Bunkyo, Fukui 910-8507, Japan

${ }^{2}$ Department of Molecular and System Pharmacology, Graduate School of Pharmaceutical

Sciences, Kyushu University, 3-1-1 Maidashi, Higashi, Fukuoka, Fukuoka 812-8582, Japan

Correspondence: Dr. Hiroshi Ikeda

Department of Human and Artificial Intelligence Systems, University of Fukui, 3-9-1 Bunkyo, Fukui 910-8507, Japan

Tel: +81(0)776 278658

Fax: +81(0)776 278420

e-mail: ikeda@synapse.his.fukui-u.ac.jp

Running title: $\alpha \beta$ meATP-induced LTP in spinal dorsal horn

The total number of pages 33 , figures 9 , tables 0 , equations 0

The total number of words in the whole manuscript 7363, abstract 199, introduction 412

Keywords: ATP, optical imaging, astrocyte, pain 


\begin{abstract}
By imaging neuronal excitation in rat spinal cord slices with a voltage-sensitive dye, we examined the role of glial cells in the $\mathrm{P} 2 \mathrm{X}$ receptor agonist, $\alpha \beta$-methylene ATP, ( $\alpha \beta$ meATP)-triggered long-term potentiation (LTP) in the dorsal horn. Bath application of $\alpha \beta$ meATP potentiated neuronal excitation in the superficial dorsal horn. The potentiation was inhibited in the presence of $\mathrm{P} 2 \mathrm{X}$ receptor antagonists, TNP-ATP, PPAADS and A-317491, and was not induced in slices taken from rats neonatally treated with capsaicin. These results suggest that $\alpha \beta$ meATP acts on $\mathbf{P} 2 \mathbf{X}$ receptors, possibly $\mathbf{P} 2 \mathbf{X}_{3}$ and/or $\mathbf{P} 2 \mathbf{X}_{\mathbf{2} / 3}$ in capsaicin-sensitive primary afferent terminals. Furthermore, the potentiation was inhibited by treatment with the glial metabolism inhibitor, monofluoro-acetic acid. Results obtained with the p38 mitogen-activated protein kinase (p38 MAPK) inhibitor, SB203580, tumor necrosis factor- $\alpha$ (TNF- $\alpha$ ), interleukin (IL)-6, and antibodies to TNF- $\alpha$ and IL-6, as well as by double immunolabeling of activated p38 MAPK with markers of astrocytes and microglia, demonstrated that $\alpha \beta$ meATP activated p38 MAPK in astrocytes, and that the presence of proinflammatory cytokines and p38 MAPK activation were necessary for the induction of $\alpha \beta$ meATP-triggered LTP. These findings indicate that glial cells contribute to the $\alpha \beta$ meATP-induced LTP, which might be part of a cellular mechanism for the induction of persistent pain.
\end{abstract}




\section{Introduction}

Peripheral inflammation or tissue injury increases the sensitivity to noxious stimuli (hyperalgesia) and/or induces pain sensation by light-touch (allodynia). Long-term potentiation (LTP) of synaptic strength in the spinal dorsal horn is now believed to be a mechanism of such persistent pain (Sandkühler \& Liu, 1998; Ma \& Zhao, 2002; Ikeda et al., 2003; Ikeda \& Murase, 2004). Adenosine triphosphate (ATP) is a major sensory neurotransmitter that activates a family of P2X receptor-modulated cation channels in the peripheral and central nervous systems (Jahr \& Jessell, 1983; Krishtal et al., 1983; Khakh et al., 2001). In hippocampal CA1 neurons, it is reported that LTP can be induced by extracellular application of ATP (Yamazaki et al., 2003), and a large number of behavioral studies have reported a contribution of spinal cord P2X receptors to hyperalgesia and allodynia (Tsuda et al., 1999; Barclay et al., 2002; McGaraughty et al., 2003; Chen et al., 2005). However, it is not known whether ATP can induce LTP in the spinal dorsal horn.

Glial cells, including microglia and astrocytes, in the spinal dorsal horn play a role in the induction and maintenance of pathological pain due to inflammation or nerve injury. The density of glial cells in the spinal cord increases after nerve injury (Garrison et al., 1994), and blocking the activation of spinal cord glial cells with fluorocitrate, an inhibitor of glial metabolic activity, prevents the induction of allodynia and hyperalgesia (Meller et al., 1994; Milligan et al., 2003). It was recently suggested that glial cell-dependent mechanisms are necessary for induction of LTP. In spinal cord slices, we have reported that a glial metabolic inhibitor blocks LTP of optically recorded neuronal excitation induced by low-frequency conditioning stimulation to the dorsal root (Ikeda \& Murase 2004). An in 
vivo study showed that disrupting the function of glial cells by a glial metabolic inhibitor blocked tetanic sciatic stimulation-induced LTP of C-fiber-evoked field potentials in the spinal dorsal horn (Ma \& Zhao, 2002). A large number of studies indicate the contribution of astrocytes to persistent pain (Coyle, 1998; Hashizume et al., 2000; DeLeo et al., 2000; Stuesse et al., 2001). Although evidence for glial contribution to the LTP in spinal dorsal horn has accumulated, the underlying mechanism is still unclear.

In this study using optical imaging with a voltage-sensitive dye, we show that extracellular application of the P2X receptor agonist, $\alpha \beta$ meATP, induces LTP of neuronal excitation in the spinal dorsal horn, and that glial cell-dependent mechanisms are involved in the LTP induction.

\section{Materials and Methods}

\section{Preparation}

All animal studies were undertaken according to protocols approved by the university animal ethics committee. Briefly, 18- to 25-day-old Wister rats were anaesthetized by diethyl ether. After performing laminectomy, the spinal cord was excised and several transverse slices (500 $\mu \mathrm{m}$ thick) with dorsal root attached were prepared from the lumbosacral enlargement. The animals were then sacrificed by an over dose of ether. Each slice was stained in a bath filled with the voltage-sensitive absorption dye, RH-482 (0.1 $\mathrm{mg} / \mathrm{ml}, 20 \mathrm{~min})$, and set in a submersion-type chamber $(0.2 \mathrm{ml})$ on an inverted microscope (IMT, Olympus, Tokyo) equipped with a 150-W halogen lamp. Slices were perfused with 
Ringer's solution containing (in mM): $124 \mathrm{NaCl}, 5 \mathrm{KCl}, 1.2 \mathrm{KH}_{2} \mathrm{PO}_{4}, 1.3 \mathrm{MgSO}_{4}, 2.4$

$\mathrm{CaCl}_{2}, 26 \mathrm{NaHCO}_{3}, 0.2$ thiourea, 0.2 ascorbic acid, and 10 glucose (oxygenated with 95\% $\mathrm{O}_{2}$ and $\left.5 \% \mathrm{CO}_{2}\right)$ at room temperature $\left(23 \pm 2^{\circ} \mathrm{C}\right)$.

\section{Optical recording}

The light absorption change, at a wavelength of $700 \pm 32 \mathrm{~nm}$, in a $0.83 \mathrm{~mm}^{2}$ area of the dorsal horn was recorded by an imaging system (Deltalon 1700, Fuji Film Co., Tokyo) with $128 \times 128$ pixel photo sensors at a frame rate of $0.6 \mathrm{~ms}$. The image of the spinal cord slice was focused on the photo sensors by an objective lens (magnification $\times 10$, numerical aperture 0.30 , Olympus). The cut end of the dorsal root attached to the slice was drawn into a glass suction electrode filled with Ringer's solution, and was stimulated by a current pulse of $2 \mathrm{~mA}$ and a duration of $0.5 \mathrm{~ms}$. The electrode was made by hand-pulling a standard Pasteur pipette over flame, and the orifice was made just to fit the dorsal root. Two silver wires, one from the inside of the pipette and another from the orifice, were connected to the stimulator. Eight single pulses were given at a constant interval of 15 s. Starting $10 \mathrm{~ms}$ before each stimulus, the image sensor took 128 consecutive frames of the light-absorption images at a sampling interval of $0.6 \mathrm{~ms}$. A reference frame taken immediately before each series of 128 frames was subtracted from the subsequent 128 frames. Eight series of difference images were averaged and stored in the system memory. The initial frame was determined by averaging the first 15 frames of the difference image and then subtracting this average from each of the 128 frames of the image data on a pixel-by-pixel basis to eliminate the effects of noise contained in the reference frame. The ratio image was then 
calculated by dividing the image data by the reference frame.

\section{Neonatal capsaicin treatment}

Postnatal day 2 rats were anaesthetized with diethyl ether and injected subcutaneously at the dorsal cervix with a capsaicin solution (50 mg/kg). Three weeks after the injection, rats were tested for one min on a hot plate $\left(65^{\circ} \mathrm{C}\right)$. While normal, untreated rats raised and licked their feet within $10 \mathrm{~s}$, successfully treated rats did not react for at least one min.

\section{Immunohistochemistry}

Slices $(500 \mu \mathrm{m})$ incubated in $\alpha \beta$ meATP for $0,10,20$, and 50 min were fixed in ice-cold $4 \%$ paraformaldehyde and sunk in $30 \%$ sucrose for $48 \mathrm{~h}$ at $4^{\circ} \mathrm{C}$. The slices were then sectioned (30 $\mu \mathrm{m})$, blocked in 3\% normal goat serum (NGS), and incubated for $48 \mathrm{~h}$ at $4^{\circ} \mathrm{C}$ in anti-phospho-p38 MAPK (1:200; Cell Signaling, Beverly, MA). In addition, antibodies directed against markers of microglia, OX42 (anti-OX42, 1:1000, Serotec, Oxford, UK) and astrocytes, glial fibrillary acidic protein (GFAP; anti-GFAP, 1:500; Boehringer-Mannheim, Indianapolis, IN) were used to identify the type of phospho-p38 MAPK-positive cells. Following incubation, tissue sections were washed and incubated for $3 \mathrm{~h}$ at room temperature in the secondary antibody solution (anti-rabbit IgG-conjugated Alexa Fluor $^{\mathrm{TM}} 488$ or anti-mouse IgG-conjugated Alexa Fluor 546, 1:1,000; Molecular Probes, Eugene, OR). The spinal cord sections were analyzed using an Axiovert 200M microscope equipped with a Zeiss LSM 510 confocal laser-scanning microscope (Carl Zeiss, Jena, Germany). 


\section{Drugs}

The RH-482 (NK-3630) dye was obtained from Nippon Kanko Shikiso (Okayama, Japan). The D-2-amino-5-phosphonovaleric acid, $\alpha \beta$-methylene ATP, TNF- $\alpha$, PPADS, A-317491, capsaicin, IL-6, monofluoroacetic acid, and SB203580 were from Sigma (St. Louis, MO). TNF- $\alpha$ and IL-6 antibodies and their control immunoglobulin G (IgG) were from R \& D systems (Minneapolis, MN).

\section{Statistics}

Results were expressed as means \pm SE. The Student's paired t-test was used to exhibit statistical differences.

\section{Results}

\section{$\alpha \beta m e A T P-i n d u c e d ~ L T P$ of neuronal excitation evoked by $C$ afferents}

We visualized gross neuronal excitation in the dorsal horn region of transverse spinal cord slices stained with the voltage-sensitive dye, RH-482 (Ikeda et al., 1998, 2004). A test stimulus, a single pulse of C fiber-activating strength to the dorsal root, evoked an optical response in spinal lamina I-III (Fig. 1A), representing the excitation of neurons throughout the thickness of the slice.

First we examined the effect of the P2X receptor agonist $\alpha \beta$ meATP on gross neuronal excitation in the spinal dorsal horn. Bath application of $\alpha \beta$ meATP $(100 \mu \mathrm{M})$ for 
50 min significantly potentiated neuronal excitation in the superficial dorsal horn elicited by a test stimulus to the dorsal root (Fig. 1A,B,C). The magnitude of the neuronal excitation gradually increased and reached $141 \pm 6 \%(n=5, p<0.01)$ of control 350 minutes after the application of $\alpha \beta$ meATP (Fig. 1D).

We then examined which of the seven P2X receptor subunits that comprise ATP-activated ion channels, contributes to $\alpha \beta$ meATP-induced long-term potentiation (LTP) of neuronal excitation. As shown in Fig. 2, TNP-ATP $(1 \mu \mathrm{M})$, a specific P2X $\mathrm{X}_{1,2,3,4}$ antagonist, PPAADS $(20 \mu \mathrm{M})$, a specific P2X $\mathrm{X}_{1,2,3,5}$ antagonist, and A-317491 (1 $\left.\mu \mathrm{M}\right)$, a recently described selective $\mathrm{P}_{2} \mathrm{X}_{3}$ and $\mathrm{P} 2 \mathrm{X}_{2 / 3}$ receptor antagonist, all blocked the induction of LTP by $\alpha \beta$ meATP (TNP-ATP, $111 \pm 4 \%, \mathrm{p}=0.3, \mathrm{n}=5$ : PPADS, $109 \pm 1 \%, \mathrm{p}=0.3, \mathrm{n}=$ 4: A-317491, $108 \pm 6 \%, p=0.9, n=5)$. Application of antagonists alone did not affect the neuronal excitation evoked by dorsal root stimulation (TNP-ATP, $104 \pm 4 \%, \mathrm{p}=0.6, \mathrm{n}=5$ : PPADS, $103 \pm 6 \%, p=0.7, n=4)$. The $\alpha \beta$ meATP-induced LTP is thus mediated primarily by the $\mathrm{P} 2 \mathrm{X}_{3}$ and/or $\mathrm{P} 2 \mathrm{X}_{2 / 3}$ receptors.

The $\mathrm{P}_{2} \mathrm{X}_{3}$ receptor is selectively expressed in capsaicin-sensitive, small-diameter, primary sensory neurons (Ueno et al., 1999; Petruska et al., 2000). To investigate whether $\alpha \beta$ meATP-induced LTP is dependent on C-fiber activity, we depleted most C fiber inputs by neonatal capsaicin treatment (Yang et al., 2003) and examined the effect of $\alpha \beta$ meATP on the optical response in slices taken from these capsaicin-treated rats. In capsaicin-treated rats, neuronal excitation evoked in the dorsal horn by high-intensity stimulation (current pulse of $2 \mathrm{~mA}$ and a duration of $0.5 \mathrm{~ms}$ ) was weaker than that in normal rats (Fig. 3A,B,C). 
To confirm that capsaicin-sensitive C-fibers were abolished in capsaicin-treated rats, we examined the effect of capsaicin on neuronal excitation. In slices taken from normal rats, bath application of $1 \mu \mathrm{M}$ capsaicin for 10 min significantly depressed neuronal excitation in the dorsal horn $(-11.8 \pm 1.4 \%, \mathrm{n}=4)$, but had no effect in slices from capsaicin-treated rats (4.3 $\pm 5.8 \%, \mathrm{n}=4, \mathrm{p}<0.05$ compared with normal rat) (Fig. 3D). These results indicate that capsaicin-treated rats lacked capsaicin-sensitive afferents (Kusudo et al. 2006). The application of $\alpha \beta$ meATP did not potentiate neuronal excitation in slices taken from the capsaicin-treated rats (Fig. 3E, $103 \pm 4 \%, p=0.5, n=4)$.

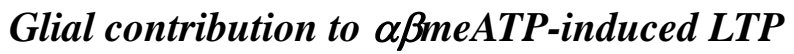

Recently, glial cells were proposed to be a key player in the induction of LTP in the central nervous system including the spinal dorsal horn (Ma \& Zhao, 2002; Ikeda \& Murase, 2004; Stellwagen \& Malenka, 2006). Therefore, we next focused on the contribution of glial cells to $\alpha \beta$ meATP-induced LTP of neuronal excitation. Treatment with the glial metabolism inhibitor, monofluoro-acetic acid $5 \mathrm{mM}$, starting $30 \mathrm{~min}$ before and continuing throughout the experiment, inhibited $\alpha \beta$ meATP-induced LTP (Fig. 4, $108 \pm 4 \%, p=0.3, n=6$ ). Monofluoro-acetic acid alone had no effect on neuronal excitation (Ikeda \& Murase, 2004). Activation of glial cells is thus necessary for $\alpha \beta$ meATP-induced LTP.

To reveal how glial cells potentiate neuronal excitation, we examined the contribution of proinflammatory cytokines TNF- $\alpha$ and IL-6, which are transmitters released from glial cells and have been implicated in the induction and maintenance of pathological 
pain (DeLeo et al., 1996;Hashizume et al., 2000;Sweitzer et al., 2001;Raghavendra et al., 2003;Milligan et al., 2001, 2003;Raghavendra et al., 2002). The $\alpha \beta$ meATP-induced LTP was blocked in slices that were pre-incubated in an antibody to rat TNF- $\alpha$ (anti-TNF- $\alpha, 10$ $\mu \mathrm{g} / \mathrm{ml}$ ) for one hour (Fig. 5A, $105 \pm 2 \%, \mathrm{p}=0.4, \mathrm{n}=4$ ). Pre-incubation in the control IgG (10 $\mu \mathrm{g} / \mathrm{ml})$ for one hour, in contrast, did not affect the LTP induction by $\alpha \beta$ meATP (Fig. 5A, $136 \pm 6 \%, p=0.9, n=4$, compared to $\alpha \beta \operatorname{meATP}$ alone). Application of TNF- $\alpha(4 \mathrm{ng} / \mathrm{ml})$ for 50 minutes potentiated neuronal excitation in the dorsal horn (Fig. 5B, $130 \pm 8 \%, p<0.05, n=4$ ). However, in slices that were pre-incubated in $\alpha \beta$ meATP for 50 min 3 hours before recording, TNF- $\alpha$ did not potentiate the neuronal excitation (Fig. 5B, $102 \pm 4 \%, p=0.6, n=4$ ).

In slices that were pre-incubated in an antibody to rat IL-6 (anti-IL6, $5 \mu \mathrm{g} / \mathrm{ml}$ ) for one hour, $\alpha \beta$ meATP-induced LTP was significantly less, especially at the late phase (Fig. 5C, $118 \pm 6 \%, p=0.2$, and $113 \pm 3 \%, p<0.01$, at 120 and 270 min after $\alpha \beta$ meATP application, respectively, $\mathrm{n}=5$, compared to $\alpha \beta$ meATP alone). Pre-incubation in the control IgG (5 $\mu \mathrm{g} / \mathrm{ml})$ for one hour did not affect the induction of LTP (Fig. 5C, $134 \pm$ $6 \%, \mathbf{p}=\mathbf{0 . 9}, \mathbf{n}=4$, compared to $\alpha \beta$ meATP alone). The application of $40 \mathrm{nM}$ IL-6 potentiated neuronal excitation in slices that were pre-incubated in TNF- $\alpha$ for 50 minutes 3 hours before recording (Fig. 5D, $128 \pm 5 \%, \mathrm{p}<0.05, \mathrm{n}=4$ ), but did not potentiate neuronal excitation in slices not pre-incubated with TNF- $\alpha$ (Fig. 5D, $97 \pm 6 \%, p=0.3, n=$ 4). From these results, it is reasonable to conclude that TNF- $\alpha$ and IL-6 contribute to $\alpha \beta$ meATP-induced LTP, and that pre-activation of the TNF- $\alpha$ receptor is necessary for the 
potentiation of neuronal excitation by IL-6.

p38 MAPK plays an important role in the induction of proinflammatory cytokines (Hua et al., 2002;Inoue et al., 2003;Chaparro-Huerta et al., 2005). We therefore examined the contribution of p38 MAPK to $\alpha \beta$ meATP-induced LTP. In the presence of $1 \mu \mathrm{M}$ SB203580, a specific inhibitor of p38 MAPK, neuronal excitation was not potentiated by $\alpha \beta$ meATP (Fig. 6A, $101 \pm 6 \%, p=0.8, n=4$ ). To confirm that the glial cells expressed activated p38 MAPK, we performed double immunolabeling for activated p38 MAPK with anti-phospho extracellular signal-regulated kinase (anti-phospho-ERK) and for either astrocytes with GFAP or microglia with anti-OX42. In the first series of experiments, single immunofluorescence labeling in spinal cord slices showed that activation of p38 MAPK is induced by $\alpha \beta$ meATP. Phospho-ERK was strongly expressed in spinal cord slices incubated in $\alpha \beta$ meATP for 10 and 20 min but not in those without $\alpha \beta$ meATP treatment (Fig. 6B-E). We also found that cells showing phospho-ERK immunofluorescence were double labeled for GFAP, but not for OX42, indicating that activated p38 MAPK was expressed in astrocytes but not in microglia (Fig. 6F,G).

\section{Mechanism of p38 MAPK activation in astrocytes}

We then examined how p38 MAPK is activated in astrocytes. It is known that MAPKs can be activated via $\mathrm{Ca}^{2+}$-permeable ionotropic receptors such as the N-methyl-D-aspartate (NMDA) receptor (Xia et al., 1996; Kawasaki et al., 1997). Therefore, we examined whether NMDA receptors contribute to $\alpha \beta$ meATP-induced LTP of the neuronal excitation. In the presence of an NMDA receptor antagonist, $\mathrm{D}(-)$-2-amino-5-phosphonopentanoic acid 
(D-AP5, $50 \mu \mathrm{M}$ ), the potentiation of neuronal excitation by $\alpha \beta$ meATP was inhibited (Fig. 7A, $102 \pm 9 \%, p=0.4, n=5)$. We also examined whether it is possible to activate p38 MAPK by directly activating NMDA receptors with NMDA. Although in neurons it is blocked by $\mathrm{Mg}^{2+}$, in glial cells activation of NMDA receptors is not affected by $\mathrm{Mg}^{2+}($ Ziak

et al., 1998, $\mathrm{Hu}$ et al., 2004; Lalo et al., 2006). In a solution containing $\mathrm{Mg}^{2+}$, the application of NMDA $(50 \mu \mathrm{M})$ for 1 min significantly potentiated neuronal excitation elicited by a test stimulus (Fig. 7B). The magnitude of neuronal excitation gradually increased and reached $154 \pm 22 \%(\mathrm{p}<0.05, \mathrm{n}=6)$ of control 120 minutes after the application of NMDA. This LTP was blocked in the presence of the p38 MAPK inhibitor, SB203580 (Fig. 7B, $96 \pm 11 \%, p=0.8, n=5$ ). These results indicate that p38 MAPK is activated via the NMDA receptor in astrocytes.

\section{Relationship between LTP induced by $\alpha \beta m e A T P$ and conditioning stimulation}

Our previous study showed that low-frequency conditioning stimulation (LFS, $2 \mathrm{~Hz}$ for 2 minutes) to the dorsal root potentiated optically-recorded neuronal excitation in the spinal dorsal horn for more that 4 hours, and the potentiation depended on glial cell activity (Ikeda and Murase, 2004). Therefore, here we examined whether LTP of neuronal excitation induced by LFS and by $\alpha \beta$ meATP share the same mechanisms. The application of $\alpha \beta$ meATP did not induce further potentiation in slices after pre induction of LTP by LFS, strongly suggesting that the two are related (Fig. 8, $134 \pm 11 \% 150$ minutes after LFS, 130 $\pm 3 \% 150$ minutes after $\alpha \beta$ meATP application, $\mathrm{p}=0.8, \mathrm{n}=3$ ). 


\section{Discussion}

In this study, we used spinal cord slices and optical imaging with a voltage-sensitive dye to record neuronal excitation in the spinal dorsal horn evoked by dorsal root stimulation. Neuronal excitation in the superficial dorsal horn was facilitated for more than 5 hours by $\alpha \beta$ meATP. We investigated on, (1) the type(s) of receptors responsible for this potentiation, (2) the contributions of glial cells and cytokines to it, (3) its relation to the NMDA receptor, and finally (4) its relation to LTP induced by low frequency conditioning stimulation.

\section{$\alpha \beta m e A T P-i n d u c e d$ LTP and pain-related LTP in the dorsal horn}

In the present study, $\alpha \beta$ meATP potentiated dorsal root-evoked neuronal excitation in the spinal dorsal horn. Electrophysiological studies report that LTP in the spinal dorsal horn is induced by repetitive high- and low-frequency conditioning stimulation to primary afferents and also by administration of natural inflammation mediators (Sandkühler \& Liu, 1998; Ma \& Zhao, 2002; Ikeda et al., 2003; Ikeda \& Murase, 2004). It is known that ATP is released not only from primary afferent terminals (Jahr \& Jessell, 1983; Krishtal et al., 1983; Khakh et al., 2001), but also from interneurons (Jo \& Schlichter, 1999) and glial cells (Anderson et al., 2004). In addition, ATP is known as a mediator of pain (Tsuda et al., 1999; Barclay et al., 2002; McGaraughty et al., 2003; Chen et al., 2005). To our knowledge, this study is the

first to demonstrate that activation of ATP receptors induces prolonged facilitation of evoked neuronal excitation in the superficial dorsal horn. That $\alpha \beta$ meATP did not induce 
facilitation once LTP was induced by low-frequency conditioning stimulation-suggests that it might share some mechanisms with LFS-induced LTP, although additional pharmacological studies are necessary to clarify this relationship. It would also be interesting to know the source(s) of endogenous ATP as well as its actions in situ.

\section{Type and location of purinergic receptors activated by $\alpha \beta m e A T P$}

It is known that P2X receptors are expressed on neurons (Jo \& Schlichter, 1999), glial cells (Anderson et al., 2004) and primary afferent terminals in the spinal dorsal horn (Cook et al., 1997; Vulchanova et al., 1997, 1998; Llewellyn-Smith \& Burnstock, 1998, Nakatsuka et al., 2003). We here showed that LTP was induced by $\alpha \beta$ meATP, a specific agonist for P2X $\mathrm{X}_{1}$, $\mathrm{P} 2 \mathrm{X}_{2}, \mathrm{P} 2 \mathrm{X}_{2 / 3}$ and $\mathrm{P} 2 \mathrm{X}_{3}$ receptors, and that it was inhibited by $\mathrm{P} 2 \mathrm{X}_{1,2,3,4}, \mathrm{P} 2 \mathrm{X}_{1,2,3,5}$, and selective $\mathrm{P} 2 \mathrm{X}_{3} / \mathrm{P} 2 \mathrm{X}_{2 / 3}$ receptor antagonists, (i.e., TNP-ATP, PPAADS, and A-317491, respectively). Therefore, $\alpha \beta$ meATP-induced LTP of evoked neuronal excitation observed in the present study is likely to be mediated by $\mathrm{P} 2 \mathrm{X}_{2 / 3}$ and/or $\mathrm{P} 2 \mathrm{X}_{3}$ receptors.

$\alpha \beta$ meATP did not induce facilitation in slices taken from neonatal capsaicin-treated rats that lack the capsaicin-sensitive, small-diameter, primary afferent sensory neurons. The absence of $\alpha \beta$ meATP-induced LTP in these slices might also be due to postsynaptic changes associated with the loss of fine afferent terminals. It is reported that $\mathrm{P}_{2} \mathrm{X}_{3}$ receptors are expressed in these neurons (Ueno et al., 1999; Petruska et al., 2000) and localized in primary afferent terminals in the dorsal horn (Cook et al., 1997; Vulchanova et al., 1997; 1998; Llewellyn-Smith \& Burnstock, 1998). It is also suggested that $\mathrm{P} 2 \mathrm{X}_{2 / 3}$ receptors exist in capsaicin-insensitive dorsal root ganglion (DRG) neurons, but not in 
capsaicin sensitive ones (Tsuda et al. 2000). It is therefore possible that $\alpha \beta$ meATP-induced LTP might be mediated by $\mathrm{P}_{2} \mathrm{X}_{3}$ receptors in capsaicin-sensitive primary afferent terminals. However, at present there is no direct evidence for the presence of $\mathrm{P}_{2} \mathrm{X}_{2 / 3}$ receptors on the capsaicin-insensitive primary afferent terminals in the dorsal horn. Thus, further investigations are necessary to determine the exact site of $\alpha \beta$ meATP action.

\section{Contribution of cytokines and astrocyte to $\alpha \beta m e A T P$-induced LTP}

The $\alpha \beta$ meATP-induced LTP of neuronal excitation was inhibited by the metabolic inhibitor, monofluoro-acetic acid, which inhibits an enzyme found in the Krebs cycle of glia but not of neurons (Paulsen et al., 1987; Hassel et al., 1992). Furthermore, $\alpha \beta$ meATP-activated p38 MAPK was expressed in astrocytes. These results suggest the contribution of astrocytes to $\alpha \beta$ meATP-induced LTP.

$\alpha \beta$ meATP-induced LTP was blocked by pre-application of an antibody to TNF- $\alpha$, and the late phase of the potentiation was inhibited by pre-application of an antibody to IL-6. TNF- $\alpha$ alone also potentiated the neuronal excitation, a phenomenon that was inhibited by pre-administration of $\alpha \beta$ meATP. IL-6 potentiated the neuronal excitation in slices pretreated with TNF- $\alpha$, but not in untreated ones. It is reported that the expression of IL-6 receptors is increased by TNF- $\alpha$ (Inoue et al., 2003). This evidence is in agreement with, and provides an explanation for our data. That is, $\alpha \beta$ meATP-induced LTP is mediated by the release of TNF- $\alpha$ and IL-6, and the release of TNF- $\alpha$ also increases the expression of IL-6 receptors that further enhances the potentiation of neuronal excitation. At present, 
however, it is unclear whether or not other proinflammatory cytokines also contribute to $\alpha \beta$ meATP-induced LTP. Further investigations are necessary to reveal the actions of cytokines on neurons and/or glial cells, especially those that directly record glial activities.

In this study, $\alpha \beta$ meATP-induced LTP was inhibited in the presence of the p38 MAPK inhibitor SB203580, and double immunofluorescence labeling showed that $\alpha \beta$ meATP-activated p38 MAPK was expressed in astrocytes, but not in microglia. These

results indicate that activation of p38 MAPK is necessary for the induction of $\alpha \beta$ meATP-induced LTP. There are a large number of reports that show proinflammatory cytokines are produced by intracellular signaling cascades triggered by p38 MAPK in glial cells (Hua et al., 2002; Inoue et al., 2003; Chaparro-Huerta et al., 2005). Therefore, it is possible that the proinflammatory cytokines that induced LTP in the present study are produced by the activation of p38 MAPK. To clarify the situation, more direct evidence is necessary concerning the activation of p38 MAPK by proinflammatory cytokines.

\section{Contribution of glial NMDA receptors to $\alpha \beta m e A T P$-induced LTP}

$\alpha \beta$ meATP-induced LTP of neuronal excitation was inhibited in the presence of the NMDA-receptor antagonist D-AP5, and a similar LTP was induced by the application of NMDA. Reports show that NMDA receptors on dorsal horn neurons are involved in LTP induction (Ikeda et al., 2003; Ma \& Zhao, 2002). Although glial cells also express NMDA receptors (Xia et al., 1996; Kawasaki et al., 1997), the contribution of glial NMDA receptors to LTP has not been reported. Our results indicated that $\alpha \beta$ meATP-induced LTP is likely induced via NMDA receptors on astrocytes, because NMDA-induced LTP was 
blocked by the inhibition of P38 MAPK activation in astrocytes following $\alpha \beta$ meATP application. We have not completely ruled out the possible contribution of NMDA-receptors on neurons.

Studies using whole-cell patch-clamp recordings showed that NMDA-evoked inward currents in glial cells are not sensitive to $\mathrm{Mg}^{2+}$, in contrast to those in neurons (Ziak et al., 1998, Hu et al., 2004; Lalo et al., 2006). In the present study, the application of NMDA in a solution containing $\mathrm{Mg}^{2+}$ facilitated neuronal excitation, further strengthening our hypothesis that $\alpha \beta$ meATP-induced LTP is likely mediated by NMDA receptors on astrocytes. Because exact membrane potential values were not measured by our optical method, however, it is uncertain whether $\alpha \beta$ meATP depolarized the membrane potential enough to remove the $\mathrm{Mg}^{2+}$ block from NMDA-receptor channels. In order to clarify this, it is necessary to measure the glial membrane potential with patch-clamp recording during the application of NMDA in a solution containing $\mathrm{Mg}^{2+}$.

In conclusion, we demonstrated that in spinal dorsal horn slices from normal rats, extracellular application of $\alpha \beta$ meATP triggered LTP of neuronal excitation via P2X receptors, possibly $\mathrm{P}_{2} \mathrm{X}_{3}$ and/or $\mathbf{P} 2 \mathrm{X}_{2 / 3}$ in capsaicin-sensitive primary afferent terminals, in a TNF $\alpha$ - and IL-6-dependent manner. The $\alpha \beta$ meATP-induced LTP was NMDA-dependent, and associated with p38 MAPK activation in astrocytes. The inhibition of p38 MAPK activation, and also the inhibition of glial metabolism, blocked the LTP induction. These results indicate the contribution of glial cells to the $\alpha \beta$ meATP-induced LTP. After the LTP induced by low-frequency conditioning stimulation to the afferent fibers, $\alpha \beta$ meATP was ineffective, the result suggesting that 
both LTPs might share the same mechanism at least in part. The possible mechanism of LTP induction by $\alpha \beta$ meATP is summarized in Fig. 9. To reveal the role of such LTP in pain sensation, experiments in vivo are surely necessary. 


\section{Acknowledgements}

Supported by grants to H.I. from the Japanese Society for Promotion of Science (JSPS), the Novartis Foundation for the Promotion of Science, the Sumitomo Foundation, the Kanae Foundation for Life and Socio-Medical Science, the Kato Memorial Bioscience Foundation, the Nakajima Foundation and the University of Fukui, by grants to K.M. from JSPS, the Yazaki Memorial Foundation for Science and Technology, and the University of Fukui, and by grants to K.I. and M.T. for Scientific Research (A) from the Ministry of Education, Science, Sports and Culture of Japan 


\section{References}

1. Anderson, C.M., Bergher, J.P., \& Swanson, R.A. (2004) ATP-induced ATP release from astrocytes. J. Neurochem., 88, 246-256.

2. Barclay, J., Patel, S., Dorn, G., Wotherspoon, G., Moffatt, S., Eunson, L., Abdel'al, S., Natt, F., Hall, J., Winter, J., Bevan, S., Wishart, W., Fox, A. \& Ganju, P. (2002) Functional downregulation of $\mathrm{P} 2 \mathrm{X} 3$ receptor subunit in rat sensory neurons reveals a significant role in chronic neuropathic and inflammatory pain. J. Neurosci., 22, 8139-8147.

3. Chaparro-Huerta. V., Rivera-Cervantes, M.C., Flores-Soto, M.E., Gomez-Pinedo, U. \& Beas-Zarate, C. (2005) Proinflammatory cytokines and apoptosis following glutamate-induced excitotoxicity mediated by p38 MAPK in the hippocampus of neonatal rats. J. Neuroimmunol., 165, 53-62.

4. Chen, Y., Li, G.W., Wang, C., Gu, Y. \& Huang, L.Y. (2005) Mechanisms underlying enhanced P2X receptor-mediated responses in the neuropathic pain state. Pain, 119, 38-48.

5. Cook, S.P. \& McCleskey, E.W. (1997) Desensitization, recovery and $\mathrm{Ca}^{2+}$-dependent modulation of ATP-gated P2X receptors in nociceptors. Neuropharmacology, 36, 1303-1308.

6. Coyle, D.E. (1998) Partial peripheral nerve injury leads to activation of astroglia and microglia which parallels the development of allodynic behavior. Glia, 23, 75-83. 
7. DeLeo, J.A., Colburn, R.W., Nichols, M. \& Malhotra, A. (1996) Interleukin-6-mediated hyperalgesia/allodynia and increased spinal IL-6 expression in a rat mononeuropathy model. J. Interferon Cytokine Res., 16, 695-700.

8. DeLeo, J.A., Rutkowski, M.D., Stalder, A.K, \& Campbell, I.L. (2000) Transgenic expression of TNF by astrocytes increases mechanical allodynia in a mouse neuropathy model. Neuroreport, 28, 599-602.

9. Garrison, C.J., Dougherty, P.M. \& Carlton, S.M. (1994) GFAP expression in lumbar spinal cord of naive and neuropathic rats treated with MK-801. Exp. Neurol., 129, 237-243.

10. Garry, E.M. \& Delaney, A., Blackburn-Munro, G., Dickinson, T., Moss, A., Nakalembe, I., Robertson, D.C., Rosie, R., Robberecht, P., Mitchell, R. \& Fleetwood-Walker, S.M. (2005) Activation of p38 and p42/44 MAP kinase in neuropathic pain: involvement of VPAC2 and NK2 receptors and mediation by spinal glia. Mol. Cell. Neurosci., 30. 523-537.

11. Hashizume, H., DeLeo, J.A., Colburn, R.W. \& Weinstein, J.N. (2000) Spinal glial activation and cytokine expression after lumbar root injury in the rat. Spine, 25, 1206-1217.

12. Hu, B., Sun, S.G. \& Tong, E.T. (2004) NMDA and AMPA receptors mediate intracellular calcium increase in rat cortical astrocytes. Acta Pharmacol. Sin., 25, 714-720.

13. Hua, L.L., Zhao, M.L., Cosenza, M., Kim, M.O., Huang, H., Tanowitz, H.B., Brosnan, C.F. \& Lee, S.C. (2002) Role of mitogen-activated protein kinases in inducible nitric 
oxide synthase and TNFalpha expression in human fetal astrocytes. J. Neuroimmunol., 126, $180-189$.

14. Hassel, B., Paulsen, R.E., Johnsen, A. \& Fonnum, F. (1992) Selective inhibition of glial cell metabolism in vivo by fluorocitrate. Brain Res., 576, 120-124.

15. Ikeda, H., Ryu, P.D., Park, J.B., Tanifuji, M., Asai, T. \& Murase, K. (1998) Optical responses evoked by single-pulse stimulation to the dorsal root in the rat spinal dorsal horn in slice. Brain Res., 812, 81-90.

16. Ikeda, H., Heinke, B., Ruscheweyh, R. \& Sandkühler, J. (2003) Synaptic plasticity in spinal lamina I projection neurons that mediatehyperalgesia. Science. 299. 1237-1240.

17. Ikeda, H. \& Murase, K. (2004) Glial nitric oxide-mediated long-term presynaptic facilitation revealed by optical imaging in rat spinal dorsal horn. J. Neurosci., 24, 9888-9896.

18. Inoue, K., Koizumi, S., Tsuda, M. \& Shigemoto-Mogami, Y. (2003) Signaling of ATP receptors in glia-neuron interaction and pain. Life Sci., 74, 189-197.

19. Jahr, C.E. \& Jessell, T.M. (1983) ATP excites a subpopulation of rat dorsal horn neurons. Nature, 304, 730-733.

20. Jin, S.X., Zhuang, Z.Y., Woolf, C.J. \& Ji, R.R. (2003) p38 mitogen-activated protein kinase is activated after a spinal nerve ligation in spinal cord microglia and dorsal root ganglion neurons and contributes to the generation of neuropathic pain. J. Neurosci., 23, 4017-4022.

21. Jo, Y.H. \& Schlichter, R. (1999) Synaptic corelease of ATP and GABA in cultured spinal neurons. Nat. Neurosci., 2, 241-245. 
22. Kawasaki, H., Morooka, T., Shimohama, S., Kimura, J., Hirano, T., Gotoh, Y. \& Nishida, E. (1997) Activation and involvement of p38 mitogen-activated protein kinase in glutamate-induced apoptosis in rat cerebellar granule cells. J. Biol. Chem. 272, 18518-1821.

23. Khakh, B.S., Burnstock, G., Kennedy, C., King, B.F., North, R.A., Seguela, P., Voigt, M. \& Humphrey, P.P. (2001) International union of pharmacology. XXIV. Current status of the nomenclatureand properties of $\mathrm{P} 2 \mathrm{X}$ receptors and their subunits. Pharmacol. Rev., 53, 107-118.

24. Krishtal, O.A., Marchenko, S.M. \& Pidoplichko, V.I. (1983) Receptor for ATP in the membrane of mammalian sensory neurones. Neurosci. Lett., 35, 41-45.

25. Kusudo, K., Ikeda, H. \& Murase, K. (2006) Depression of presynaptic excitation by the activation of vanilloid receptor 1 in the rat spinal dorsal horn revealed by optical imaging. Mol. Pain, 2:8.

26. Lalo, U., Pankratov, Y., Kirchhoff, F., North, R.A., Verkhratsky, A. (2006) NMDA receptors mediate neuron-to-glia signaling in mouse cortical astrocytes. J. Neurosci., 26, 2673-2683.

27. Llewellyn-Smith, I.J. \& Burnstock, G. (1998) Ultrastructural localization of P2X3 receptors in rat sensory neurons. Neuroreport, 9, 2545-2550.

28. Ma, J.Y. \& Zhao, Z.Q. (2002) The involvement of glia in long-term plasticity in the spinal dorsal horn of the rat. Neuroreport, 13, 1781-1784.

29. McGaraughty, S., Wismer, C.T., Zhu, C.Z., Mikusa, J., Honore, P., Chu, K.L., Lee, C.H., Faltynek, C.R. \& Jarvis, M.F. (2003) Effects of A-317491, a novel and selective 
$\mathrm{P} 2 \mathrm{X} 3 / \mathrm{P} 2 \mathrm{X} 2 / 3$ receptor antagonist, on neuropathic, inflammatory and chemogenic nociception following intrathecal and intraplantar administration. Br. J. Pharmacol., 140, $1381-1388$.

30. Meller, S.T., Dykstra, C., Grzybycki, D., Murphy, S. \& Gebhart, G.F. (1994) The possible role of glia in nociceptive processing and hyperalgesia in the spinal cord of the rat. Neuropharmacology, 33, 1471-1478.

31. Milligan, E.D., O'Connor, K.A., Nguyen, K.T., Armstrong, C.B., Twining, C., Gaykema, R.P., Holguin, A., Martin, D., Maier, S.F. \& Watkins, L.R. (2001) Intrathecal HIV-1 envelope glycoprotein gp120 induces enhanced pain states mediated by spinal cord proinflammatory cytokines. J. Neurosci., 21. 2808-2819.

32. Milligan, E.D., Twining, C., Chacur, M., Biedenkapp, J., O'Connor, K., Poole, S., Tracey, K., Martin, D., Maier, S.F. \& Watkins, L.R. (2003) Spinal glia and proinflammatory cytokines mediate mirror-image neuropathic pain in rats. J. Neurosci ., 23, 1026-1040.

33. Nakatsuka, T., Tsuzuki, K., Ling, J.K., Sonobe, H., Gu, J.G. (2003) Distinct roles of P2X receptors modulating glutamate release at primary sensory synapses in rat spinal cord. J. Neurophysiol., 89, 3243-3252.

34. Paulsen, R.E., Contestabile, A., Villani, L. \& Fonnum, F. (1987) An in vivo model for studying function of brain tissue temporarily devoid of glial cell metabolism: the use of fluorocitrate. J. Neurochem., 48, 1377-1385. 
35. Petruska, J.C., Cooper, B.Y., Gu, J.G., Rau, K.K. \& Johnson, R.D. (2000) Distribution of $\mathrm{P} 2 \mathrm{X} 1, \mathrm{P} 2 \mathrm{X} 2$, and $\mathrm{P} 2 \mathrm{X} 3$ receptor subunits in rat primary afferents: relation to population markers and specific cell types. J. Chem. Neuroanat., 20, 141-162.

36. Raghavendra, V., Rutkowski, M.D. \& DeLeo, J.A. (2002) The role of spinal neuroimmune activation in morphine tolerance/hyperalgesia in neuropathic and sham-operated rats. J. Neurosci., 22, 9980-9989.

37. Raghavendra, V., Tanga, F., Rutkowski, M.D. \& DeLeo, J.A. (2003) Anti-hyperalgesic and morphine-sparing actions of propentofylline following peripheral nerve injury in rats: mechanistic implications of spinal glia and proinflammatory cytokines. Pain, 104, 655-664.

38. Sandkühler, J. \& Liu, X. (1998) Induction of long-term potentiation at spinal synapses by noxious stimulation or nerve injury. Eur. J. Neurosci., 10, 2476-2480.

39. Stellwagen, D. \& Malenka, R.C. (2006) Synaptic scaling mediated by glial TNF-alpha. Nature, 440, 1054-1059.

40. Stuesse, S.L., Crisp, T., McBurney, D.L., Schechter, J.B., Lovell, J.A. \& Cruce, W.L. (2001) Neuropathic pain in aged rats: behavioral responses and astrocytic activation. Exp. Brain Res., 137, 219-227.

41. Svensson, C.I., Marsala, M., Westerlund, A., Calcutt, N.A., Campana, W.M., Freshwater, J.D., Catalano, R., Feng, Y., Protter, A.A., Scott, B. \& Yaksh, T.L. (2003) Activation of p38 mitogen-activated protein kinase in spinal microglia is a critical link in inflammation-induced spinal pain processing. J. Neurochem., 86, 1534-1544. 
42. Sweitzer, S., Martin, D. \& DeLeo, J.A. (2001) Intrathecal interleukin-1 receptor antagonist in combination with soluble tumor necrosis factor receptor exhibits an anti-allodynic action in a rat model of neuropathic pain. Neuroscience, 103, 529-539.

43. Tsuda, M., Ueno, S., Inoue, K. (1999) Evidence for the involvement of spinal endogenous ATP and P2X receptors in nociceptive responses caused by formalin and capsaicin in mice. Br. J. Pharmacol., 128, 1497-1504.

44. Tsuda, M., Koizumi, S., Kita, A., Shigemoto, Y., Ueno, S., Inoue, K. (2000) Mechanical allodynia caused by intraplantar injection of P2X receptor agonist in rats: involvement of heteromeric $\mathrm{P} 2 \mathrm{X}_{2 / 3}$ receptor signaling in capsaicin-insensitive primary afferent neurons. J. Neurosci., 20, 1-5.

45. Tsuda, M., Mizokoshi, A., Shigemoto-Mogami, Y., Koizumi, S. \& Inoue, K. (2004) Activation of p38 mitogen-activated protein kinase in spinal hyperactive microglia contributes to pain hypersensitivity following peripheral nerve injury. Glia, 45, 89-95.

46. Ueno, S., Tsuda, M., Iwanaga, T. \& Inoue, K. (1999) Cell type-specific ATP-activated responses in rat dorsal root ganglion neurons. Br. J. Pharmacol., 126, 429-436.

47. Vulchanova, L., Riedl, M.S., Shuster, S.J., Buell, G., Surprenant, A., North, R.A. \& Elde, R. (1997) Immunohistochemical study of the P2X2 and P2X3 receptor subunits in rat and monkey sensory neurons and their central terminals. Neuropharmacology, 36, 1229-1242.

48. Vulchanova, L., Riedl, M.S., Shuster, S.J., Stone, L.S., Hargreaves, K.M., Buell, G., Surprenant, A., North, R.A. \& Elde, R. (1998) P2X3 is expressed by DRG neurons that terminate in inner lamina II. Eur, J. Neurosci., 10, 3470-3478. 
49. Xia, Z., Dudek, H., Miranti, C.K. \& Greenberg, M.E. (1996) Calcium influx via the NMDA receptor induces immediate early gene transcription by a MAP kinase/ERK-dependent mechanism. J. Neurosci., 16, 5425-5436.

50. Yamazaki, Y., Kaneko, K., Fujii, S., Kato, H. \& Ito, K. (2003) Long-term potentiation and long-term depression induced by local application of ATP to hippocampal CA1 neurons of the guinea pig. Hippocampus, 13, 81-92.

51. Yang, K., Furue, H., Fujita, T., Kumamoto, E. \& Yoshimura, M. (2003) Alterations in primary afferent input to substantia gelatinosa of adult rat spinal cord after neonatal capsaicin treatment. J. Neurosci. Res., 74, 928-933.

52. Ziak, D., Chvatal, A. \& Sykova, E. (1998) Glutamate-, kainate- and NMDA-evoked membrane currents in identified glial cells in rat spinal cord slice. Physiol. Res., 47, 365-375. 


\section{Figure Legends}

\section{Fig. 1}

LTP of the neuronal excitation in the spinal dorsal horn by $\alpha \beta$ meATP. A, Optical responses elicited by a single-pulse test stimulation to the dorsal root immediately before (left images) and 300 min after (right images) $\alpha \beta$ meATP application. Images were taken from the area indicated by the black rectangle frame in the schematic drawing of a transverse slice in $\mathbf{C}$. The times after the test stimuli are indicated to the left of the images. The percent change in light absorption is depicted using simulated color as described in the color bar. B, Superimposed time courses of optical responses in the pixels before (solid lines) and after (dashed lines) $\alpha \beta$ meATP application. The time points are indicated by numbers 1 and 2 in $\mathbf{D}$. The traces are the responses of $3 \times 2$ pixels (at a 5-pixel interval on the image sensor) within the red rectangle on the schematic drawing in $\mathbf{C}$. The stimulus pulses were given at the time indicated by the filled triangle. $\mathbf{C}$, Schematic drawing of a transverse spinal cord slice (left), and the spatial averages of time courses before (solid line) and after (dashed line) $\alpha \beta$ meATP application (right). Each time course was an average of 16 optical responses recorded by $4 \times 4$ pixels on the image sensor in the dorsal horn indicated by the black rectangle in the schematic drawing. We here call it the spatial average. $\mathbf{D}$, The mean magnitudes of the optical responses with $\alpha \beta$ meATP application (filled circles) and without (open circles). Magnitude of each response was obtained by the temporal average during $4.8 \mathrm{~ms}$ period after the onset of response which is indicated by vertical lines in $\mathrm{C}$. Since the value is the temporal average of spatially-averaged time course, 
we here call it the spatiotemporal average. The value was then normalized to the base line values before the application of $\alpha \beta$ meATP. The mean magnitudes, as well as the standard errors, of the normalized spatiotemporal average values obtained in five slices with and without $\alpha \beta$ meATP application were illustrated in the graph. The horizontal bar indicates the time when $\alpha \beta$ meATP was applied. Graphs in the following figures represent such mean magnitudes and standard errors as well.

\section{Fig. 2}

Effect of various P2X receptor antagonists on the $\alpha \beta$ meATP-induced LTP. LTP of neuronal excitation in the spinal dorsal horn, induced by $\alpha \beta$ meATP, was inhibited in the presence of a specific $\mathrm{P} 2 \mathrm{X}_{1,2,3,4}$ receptor antagonist, TNP-ATP (A), a specific $\mathrm{P}_{2} \mathrm{X}_{1,2,3,5}$ receptor antagonist, PPADS (B), and a specific $\mathrm{P}_{2} \mathrm{X}_{3}$ and P2X $\mathrm{X}_{2 / 3}$ receptor antagonist, A-317491 (C). The optical responses were taken at every 8 min in $4-5$ slices. The horizontal bars indicate the time when drugs were applied.

\section{Fig. 3}

The effect of $\alpha \beta$ meATP on neuronal excitation in slices taken from capsaicin-treated rats. A, B, An example of optical responses elicited by single-pulse test stimulations to the dorsal root in a slice taken from a normal rat (A) and a capsaicin-treated rat (B). C, Spatial distributions of the optical responses shown in A (normal rat, black line) and $\mathbf{B}$ (capsaicin-treated rat, blue line) along a dorso-ventral line in the schematic drawing of transverse slices in $\mathbf{A}$ and $\mathbf{B}$. The depth of the white matter and the substantia gelatinosa 
are indicated by WM and SG, respectively. D, An example of superimposed time courses of optical responses in a slice taken from a normal rat (upper traces) and from a capsaicin-treated rat (lower traces) before capsaicin application (black lines) and after (red lines). E, The upper panel indicates superimposed time courses of optical responses in a slice taken from a capsaicin-treated rat before $\alpha \beta$ meATP application (black line) and after (red line). The time points are indicated by numbers 1 and 2 in the lower panel. The lower panel indicates the mean magnitudes of the optical responses taken at every 8 min in four slices. The horizontal bar indicates the time when $\alpha \beta$ meATP was applied.

\section{Fig. 4}

Contribution of glial cells to the $\alpha \beta$ meATP-induced LTP. LTP of neuronal excitation in the spinal dorsal horn by $\alpha \beta$ meATP was inhibited in the presence of the glial metabolism inhibitor, monofluoro-acetic acid (MFA). Upper panel indicates superimposed time courses of optical responses before (solid lines) and after (dashed lines) $\alpha \beta m e A T P$ application. The time points are indicated by numbers 1 and 2 in the lower panel. The lower panel shows the mean magnitudes of optical responses obtained at every 8 min in six slices. The horizontal bar indicates the time when $\alpha \beta$ meATP was applied.

\section{Fig. 5}

Contribution of TNF- $\alpha$ and IL-6 to $\alpha \beta$ meATP-induced LTP in the spinal dorsal horn. A, $\alpha \beta$ meATP-induced LTP was not observed in four slices that were pre-incubated with 
antibody to rat TNF- $\alpha$ (filled circles), while it was present in four slices that were pre-incubated with the control IgG (open circles). The horizontal bar indicates the time when $\alpha \beta$ meATP was applied. B, TNF- $\alpha$ facilitated neuronal excitation of the spinal dorsal horn in four control slices (filled circles), but not in four slices that were incubated in $\alpha \beta$ meATP before recording (open circles). The horizontal bar indicates the time when TNF- $\alpha$ was applied. $\mathbf{C}, \alpha \beta$ meATP-induced LTP was significantly less in five slices that were incubated in antibody to rat IL-6 before recording (filled circles), while it was present in four slices that were pre-incubated with the control IgG (open circles). The horizontal bar indicates the time when $\alpha \beta$ meATP was applied. D, IL-6 facilitated the neuronal excitation in four slices that were pre-incubated in TNF- $\alpha$ (open circles), but not in four slices without pre-application of TNF- $\alpha$ (filled circles). The horizontal bar indicates the time when IL-6 was applied.

\section{Fig. 6}

Contribution of p38 MAPK to the $\alpha \beta$ meATP-induced LTP. A, $\alpha \beta$ meATP-induced LTP was inhibited in the presence of a specific p38 MAPK inhibitor, SB203580, in four slices. The horizontal bar indicates the time when $\alpha \beta$ meATP was applied. Upper panel indicates the superimposed time courses of optical responses before (solid lines) and after (dashed lines) $\alpha \beta$ meATP application. The time points are indicated by numbers 1 and 2 in the lower panel. B-E, Immunofluorescence labeling with anti-phospho-p38 MAPK (green) in a spinal cord slice without $\alpha \beta$ meATP incubation (B) and incubated in $\alpha \beta$ meATP for 10 min 
(C), $20 \mathrm{~min}$ (D), and $50 \mathrm{~min}$ (E). F, G, Double immunofluorescence labeling with anti-phospho-p38 MAPK (green) and with either anti-GFAP (F, red), a marker of astrocytes, or anti-OX42 (G, red), a marker of microglia. Most phospho-p38 MAPK-positive cells are double labeled (yellow) with GFAP (F).

\section{Fig. 7}

Contribution of NMDA receptors to the $\alpha \beta$ meATP-induced LTP. A, The LTP of neuronal excitation in the spinal dorsal horn induced by $\alpha \beta$ meATP was inhibited in the presence of the NMDA receptor antagonist, D-AP5 in five slices. The horizontal bar indicates the time when $\alpha \beta$ meATP was applied. B, Neuronal excitation was potentiated by a one min application of NMDA in six slices (filled circles) and the facilitation was not induced in the presence of the p38 MAPK inhibitor, SB203580, in five slices (open circles). Arrow indicates the time when NMDA was administered.

\section{Fig. 8}

Effect of $\alpha \beta$ meATP given after low-frequency conditioning stimulation (LFS). $\alpha \beta$ meATP that was applied after the induction of facilitation by LFS did not induce further facilitation of neuronal excitation in the spinal dorsal horn in three slices. Arrow indicates the time when LFS was applied. Horizontal bar indicates the time when $\alpha \beta$ meATP was applied.

\section{Fig. 9}

Possible mechanism of LTP induction. Activation of presynaptic ATP receptors on fine 
afferent fiber terminals, possibly of the P2X3 type, leads to facilitation of the afferent synaptic transmission. Cytokines TNF- $\alpha$ and IL-6 are necessary for the induction of facilitation. The sources and sites of action of these cytokines are yet to be identified. We propose that the activation of p38MAPK in astrocytes is initiated by the activation of NMDA receptors, which may release cytokines that facilitate the LTP. Low-frequency conditioning stimulation to afferent fibers may trigger the whole event by releasing glutamate and ATP. 


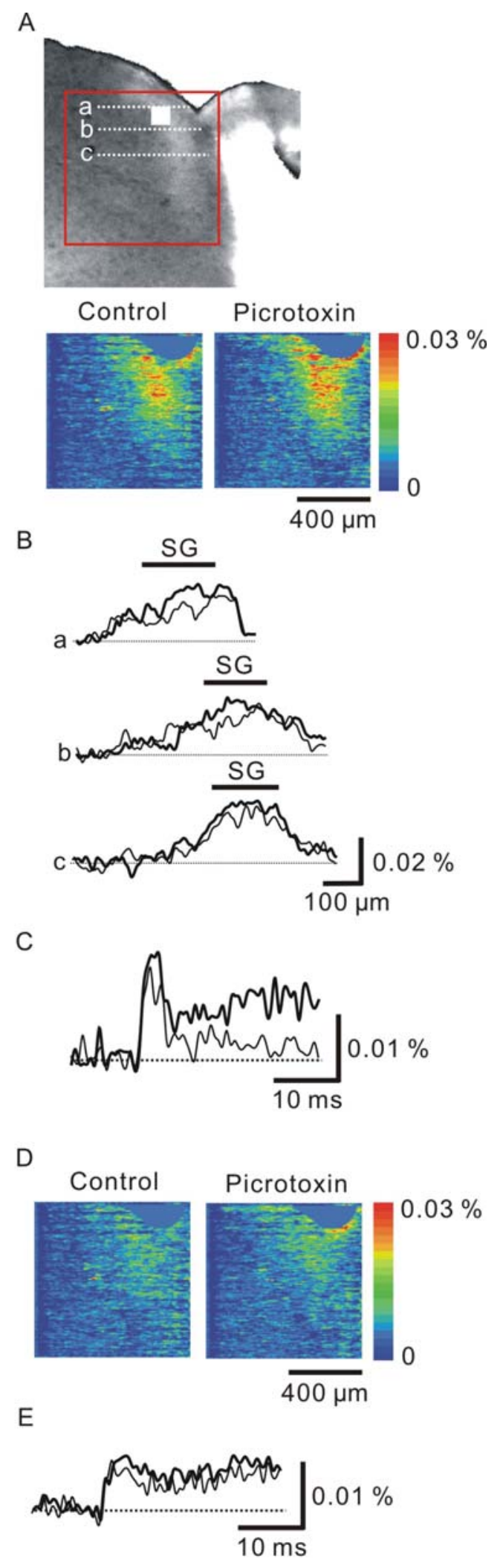


A

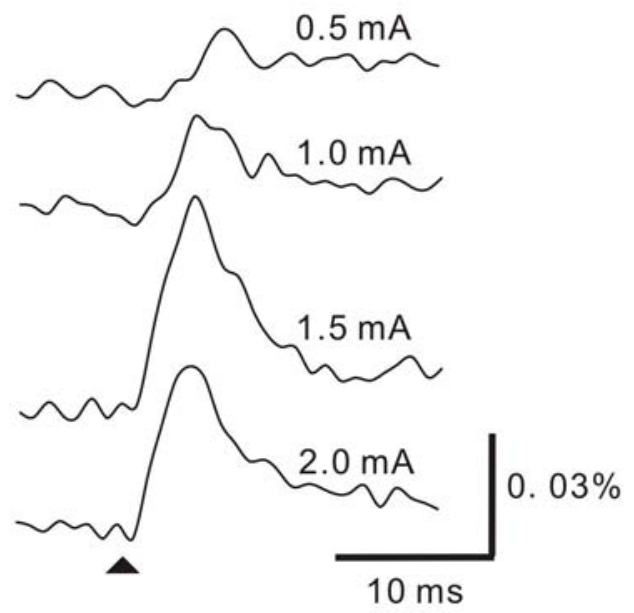

B

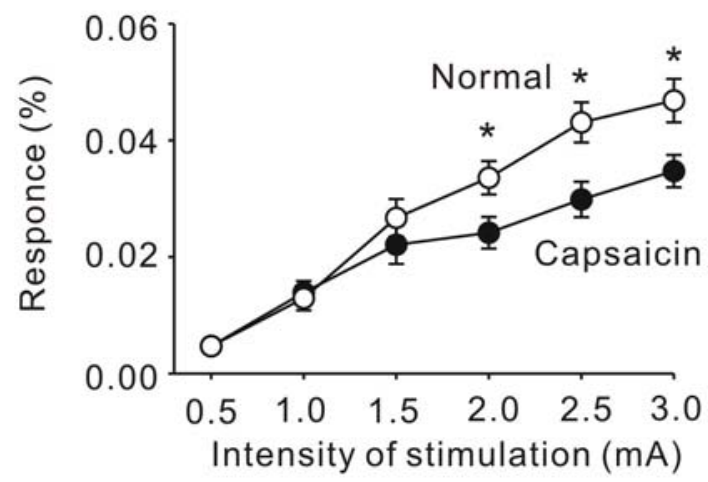

- Control 
A

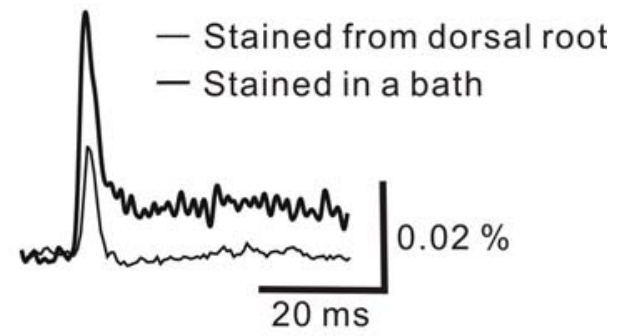

B

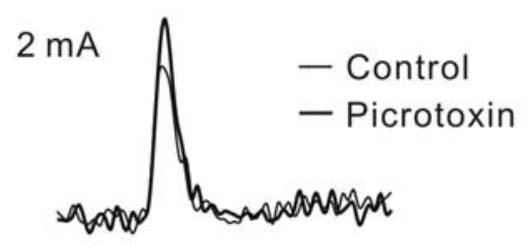

$1 \mathrm{~mA}$

Fig. 3 
A

Picrotoxin in D-AP5\&CNQX

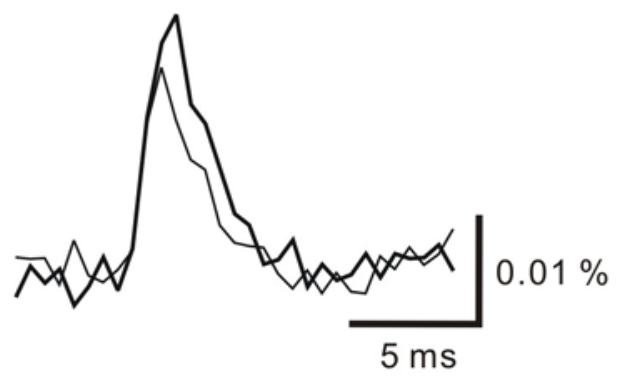

B Picrotoxin in D-AP5\&CNQX\&MFA

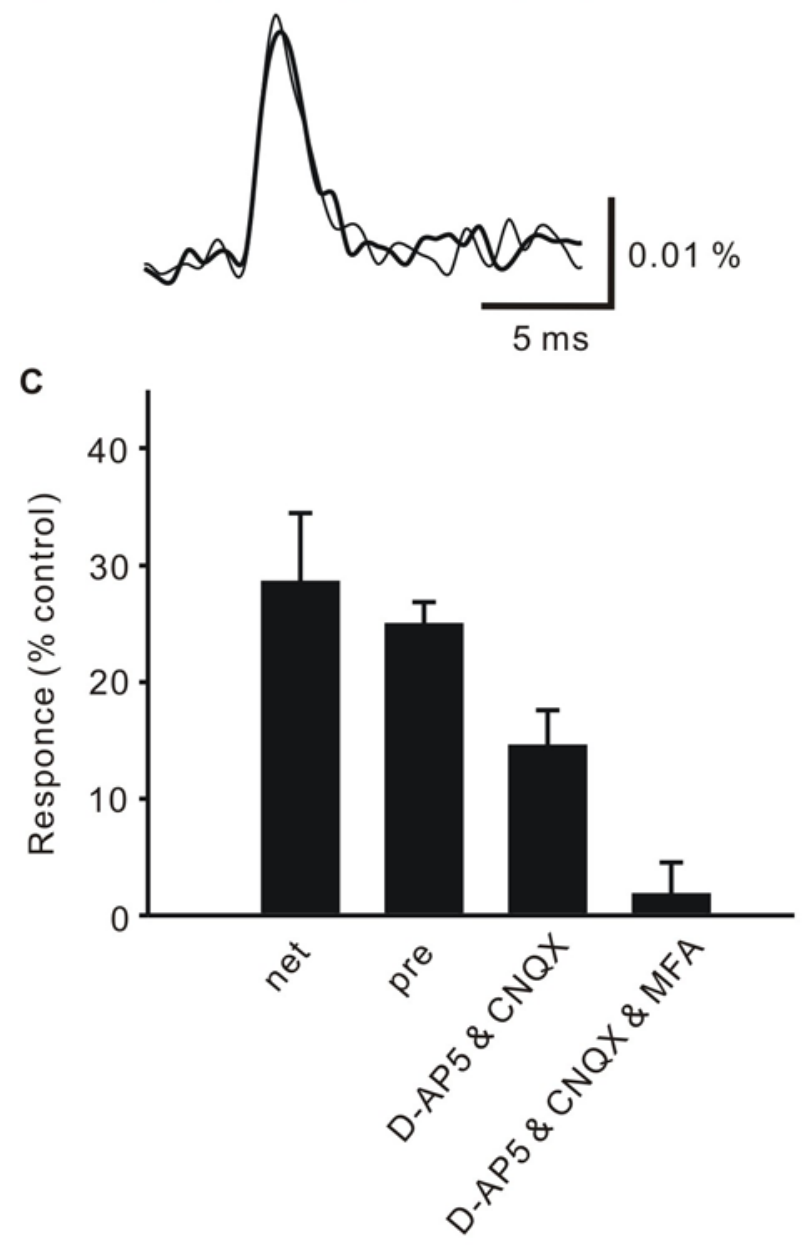

Fig. 4 
A CNQX

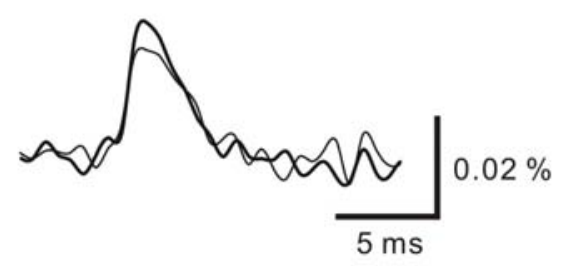

B D-AP5\&CNQX

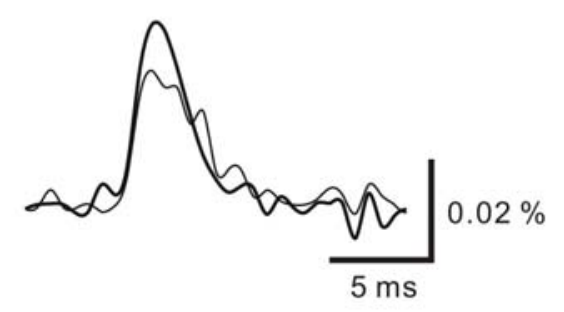

C
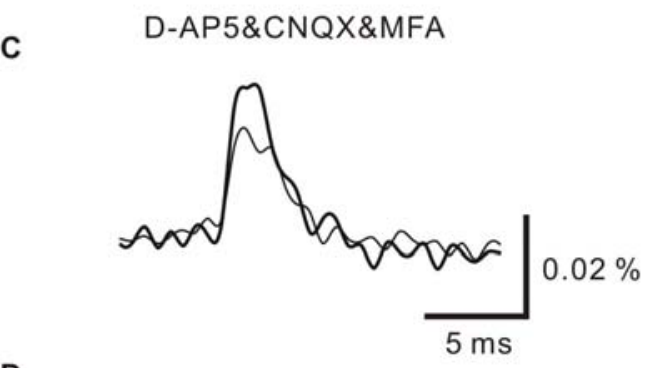

D

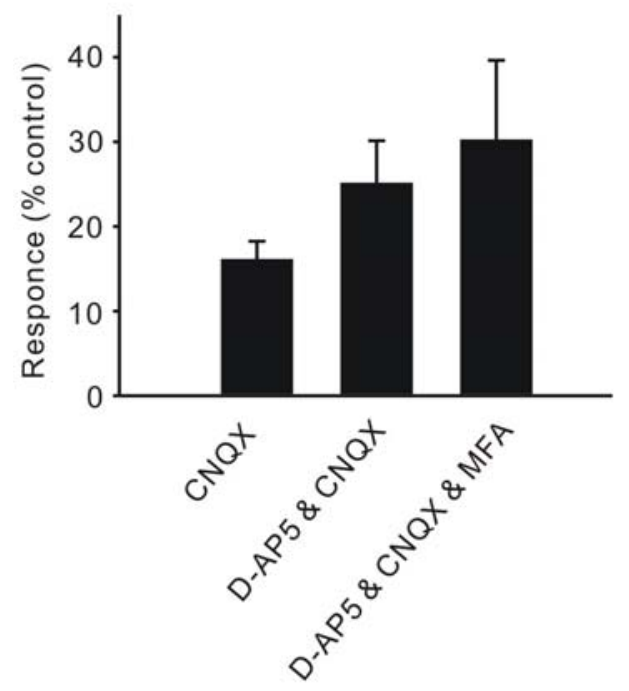

Fig. 5 


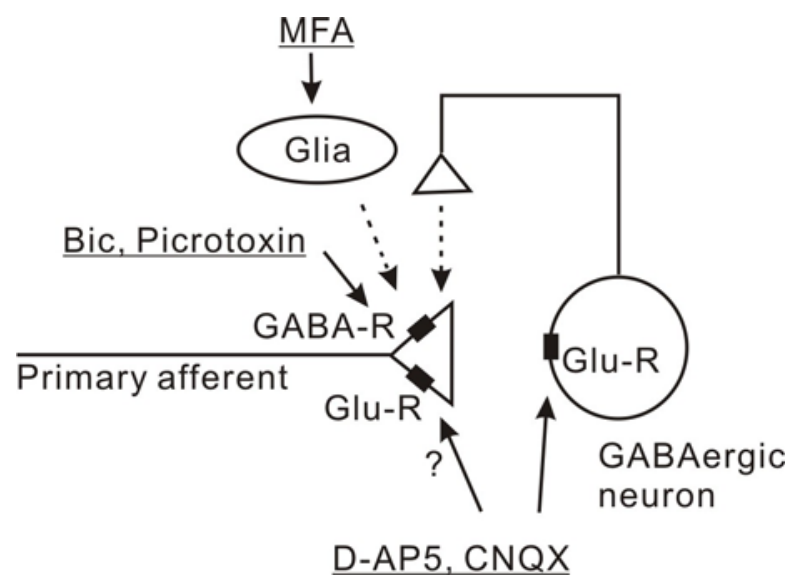


